OPEN ACCESS

Edited by:

Wellison J. S. Diniz,

Auburn University, United States

Reviewed by:

Kara Thornton,

Utah State University, United States

Min Du,

Washington State University,

United States

*Correspondence:

Wei Guo

wguo2@wisc.edu

Specialty section:

This article was submitted to

Livestock Genomics,

a section of the journal

Frontiers in Genetics

Received: 16 July 2021

Accepted: 23 December 2021

Published: 31 January 2022

Citation:

Liu Y, Ding Q, Halderson SJ, Arriola Apelo SI, Jones AK, Pillai SM, Hoffman ML, Reed S, Govoni KE, Zinn SA and Guo W (2022) Maternal

Overnutrition During Gestation in Sheep Alters Autophagy Associated

Pathways in Offspring Heart.

Front. Genet. 12:742704.

doi: 10.3389/fgene.2021.742704

\section{Maternal Overnutrition During Gestation in Sheep Alters Autophagy Associated Pathways in Offspring Heart}

Yang Liu ${ }^{1}$, Qiyue Ding ${ }^{1}$, Steven J. Halderson ${ }^{1}$, Sebastian I. Arriola Apelo ${ }^{1}$, Amanda K. Jones ${ }^{2}$, Sambhu M. Pillai ${ }^{2}$, Maria L. Hoffman ${ }^{2}$, Sarah Reed ${ }^{2}$, Kristen E. Govoni $^{2}$, Steven A. Zinn ${ }^{2}$ and Wei Guo ${ }^{1 *}$

${ }^{1}$ Department of Animal and Diary Sciences, University of Wisconsin-Madison, Madison, WI, United States, ${ }^{2}$ Department of Animal Science, University of Connecticut, Storrs, CT, United States

Poor maternal nutrition during gestation can negatively affect offspring growth, development, and health pre- and post-natally. Overfeeding during gestation or maternal obesity $(\mathrm{MO})$ results in altered metabolism and imbalanced endocrine hormones in animals and humans which will have long-lasting and detrimental effects on offspring growth and health. In this study, we examined the effects of overnutrition during gestation on autophagy associated pathways in offspring heart muscles at two gestational and one early postnatal time point ( $n=5$ for treated and untreated male and female heart respectively at each time point). Two-way ANOVA was used to analyze the interaction between treatment and sex at each time point. Our results revealed significant interactions of maternal diet by developmental stages for offspring autophagy signaling. Overfeeding did not affect the autophagy signaling at mid-gestation day 90 (GD90) in both male and female offspring while the inflammatory cytokines were increased in GD90 MO male offsrping; however, overfeeding during gestation significantly increased autophagy signaling, but not inflammation level at a later developmental stage (GD135 and day 1 after birth) in both males and females. We also identified a sexual dimorphic response in which female progeny were more profoundly influenced by maternal diet than male progeny regardless of developmental stages. We also determined the cortisol concentrations in male and female hearts at three developmental stages. We did not observe cortisol changes between males and females or between overfeeding and control groups. Our exploratory studies imply that $\mathrm{MO}$ alters autophagy associated pathways in both male and female at later developmental stages with more profound effects in female. This finding need be confirmed with larger sample numbers in the future. Our results suggest that targeting on autophagy pathway could be a strategy for correction of adverse effects in offspring of over-fed ewes.

Keywords: poor maternal nutrition, maternal obesity, developmental programming, autophagy, sheep, heart muscle 


\section{INTRODUCTION}

Developmental programming, also known as fetal programming, occurs during in utero life whilst the fetus is developing (Widdowson and McCance, 1975). During this specific window of development when the fetus is especially vulnerable, exposure of the fetus to an unfavorable uterine environment such as poor nutrition or hormonal perturbations may lead to retarded offspring growth and short- and long-term health implications (Barker et al., 1993; Barker, 2007; Gluckman et al., 2008). Nutrition is one of the major intrauterine environmental factors that can reprogram fetal growth and development during gestation in many species such as cattle, swine and sheep (Bell and Ehrhardt, 2002). Both maternal under- and over-nutrition can lead to intrauterine growth restriction, reduced birth weight, increased fetal and neonatal mortality, and altered postnatal growth rate, decreased carcass quality, feed efficiency, and negative health effects in animals and humans (Barker and Clark, 1997; Godfrey and Barker, 2001; Bell and Ehrhardt, 2002; Wallace et al., 2003; Wu et al., 2006; Ford and Long, 2011; Du et al., 2013; Reed et al., 2014).

Due to the health-associated risk factors, obesity has become a major health issue worldwide and is comorbid with increased global rates of a variety of chronic conditions including heart disease, diabetes, hypertension, elevated cholesterol, stroke, heart failure, cancers, and arthritis during early life and in adulthood (Malnick and Knobler, 2006). Further, the prevalence of maternal obesity (MO) in the United States is high and increasing (Ogden et al., 2007; Flegal et al., 2010). Nearly one-third of women are obese at child-bearing age (Caballero, 2003; Boney et al., 2005; Flegal et al., 2012; Zambrano and Nathanielsz, 2013). MO induces adverse effects on both maternal health and fetal growth and development, which can result in harmful, and persistent effects in offspring (Friedrich, 2002; Sullivan et al., 2011). Human epidemiological studies have shown that MO triggers cardiac remodeling and increases risks of offspring heart disease later in life (Reynolds et al., 2013; Gaillard, 2015). Using sheep as a model, our group revealed that MO impaired fetal cardiomyocyte contractile function by altering myofilament protein composition and disrupting calcium homeostasis through altered intracellular calcium handling signaling (Wang et al., 2019). Other research groups found that over-nutrition and MO alters the JUN N-terminal kinase (JNK)-insulin receptor substrate (IRS)-1 signaling cascade and cardiac function in the fetal heart (Wang et al., 2010) and induces fibrosis in fetal myocardium of sheep (Huang et al., 2010). Knowledge gained from the past decades has shown that energy imbalance and hormonal dysregulation are widely accepted etiological mechanisms underpinning obesity that are tightly regulated by autophagy (Zhang et al., 2018). In this study, we aimed at understanding whether MO-induced cardiac dysfunction is associated with altered autophagy signaling. We used a previously characterized MO sheep model (Pillai et al., 2017) to determine whether autophagy associated cell signaling pathways are changed in fetuses and neonates at different developmental stages in response to MO.

\section{METHODS AND MATERIALS}

\section{Animals}

All animal procedures were reviewed and approved by the University of Connecticut Institutional Animal Care and Use Committee (A13059). Animal procedures and complete experimental design details were described previously (Pillai et al., 2017). Briefly, multiparous Western White-faced ewes $(n=36)$ were estrus synchronized using a progesterone controlled intravaginal drug release device (Easi-Breed CIDR Sheep Insert, Zoetis Inc., Parsippany, NJ), followed by a single i. $\mathrm{m}$. injection of prostaglandin $\mathrm{F}_{2}$ alpha (Lutalyse, $5 \mathrm{mg} / \mathrm{ml}$; Zoetis, Inc.). Ewes were bred to 1 of 4 related Dorset rams. A rump mark received by the ewe was considered as Day 0 of pregnancy. After 20 days, ewes were then housed in individual pens. Pregnant ewes at gestation day $30.2 \pm 0.2$ were fed either a control $(100 \% \mathrm{NRC}$; CON; $n=17$ ) or over-fed diet ( $140 \%$ NRC; MO; $n=19$ ) based on National Research Council (NRC) requirements for total digestible nutrients (TDN, National Research Council, 1985). Ewes at gestation day 90 ( $n=6 \mathrm{CON}, 6 \mathrm{MO})$ or 135 ( $n=6 \mathrm{CON}$; $7 \mathrm{MO}$ ) were euthanized by an intravenous injection of Beuthanasia-D Special (Merck Animal Health; Summit, NJ) containing $390 \mathrm{mg} / \mathrm{ml}$ sodium pentobarbital and $50 \mathrm{mg} / \mathrm{ml}$ phenytoin based on body weight, and exsanguinated. A hysterectomy was performed to remove the uterus and all fetuses for fetal sample collection. A subset of ewes ( $n=5 \mathrm{CON} ; 6 \mathrm{MO})$ were allowed to give birth. Lambs were nursed for up to $24 \mathrm{~h}$, weighed, euthanized with an i., v. overdose of Beuthanasia-D Special (390 ng/ $\mathrm{ml}$ sodium pentobarbital and $50 \mathrm{mg} / \mathrm{ml}$ phenytoin based on body weight), and exsanguinated (Pillai et al., 2017; Martin et al., 2019; Gauvin et al., 2020).

\section{Sample Collection}

To obtain fetal organs, a mid-ventral incision extending from the thoracic cavity to the lower abdominal cavity was made. Heart was excised from each offspring $(n=5$ each from control and treatment group at different developmental stages). The heart was weighed and heart length and width were measured. Then heart tissues were snap-frozen in liquid nitrogen and stored at $-80^{\circ} \mathrm{C}$ freezer until analyzed.

\section{Protein Sample Preparations}

Ventricles of offspring sheep heart were weighed and approximately $50 \mathrm{mg}$ was homogenized with a glass tissue grinder in $1 \mathrm{ml}$ of Urea-Thiourea Sample Buffer [8 M urea, $2 \mathrm{M}$ thiourea, $75 \mathrm{mM}$ DTT, 3\% SDS, $0.05 \%$ bromophenol blue, and $0.05 \mathrm{M}$ Tris (pH6.8)] as described previously (Guo et al., 2012; Wang et al., 2019). After samples were completely dissolved, the protein samples were transferred to a $1.5 \mathrm{ml}$ centrifugation tube, and heated to $60^{\circ} \mathrm{C}$ for $10 \mathrm{~min}$. The protein samples were then centrifuged at $16,000 \mathrm{rpm}$ for $10 \mathrm{~min}$ at $4^{\circ} \mathrm{C}$. The supernatants were transferred to a new $1.5 \mathrm{ml}$ centrifugation tube and aliquoted and stored at $-80^{\circ} \mathrm{C}$ for later analysis.

\section{Western Blotting}

Total protein was separated by SDS-PAGE for $1.5 \mathrm{~h}$ with 120 voltage and transferred onto a PVDF membrane (Bio-Rad, Hercules, CA, Catalog \#1620177) using Bio-Rad Trans-Blot ${ }^{\circledR}$ Turbo $^{\mathrm{TM}}$ Transfer System (7 min with standard protocol 
A



p38 MAPK $--0-140 \mathrm{KD}$ p-p38 MAPK $-(-\infty-\infty-\infty \quad-\infty \mathrm{KD}$

GAPDH $===2-\infty \ldots+m$ KD

H

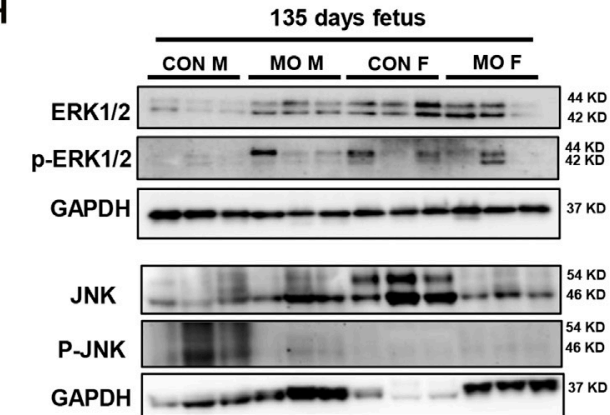

P38 MAPK $=10$

P-p38 MAPK - _ _

GAPDH $=37 \mathrm{KD}$

0

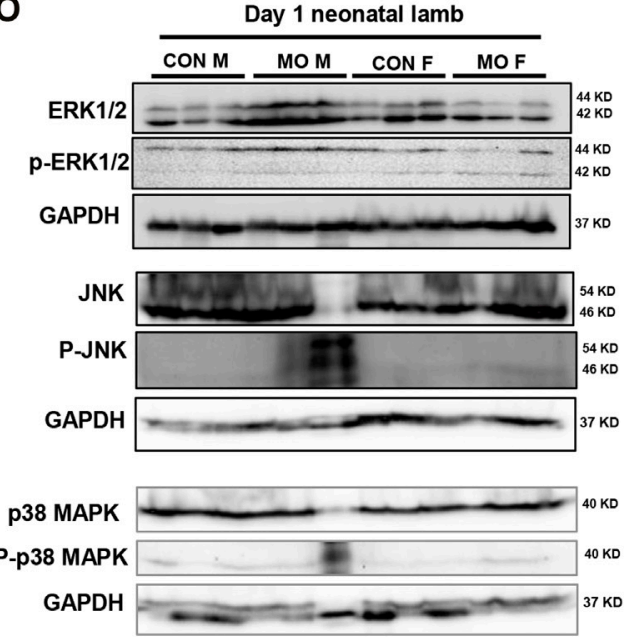

B

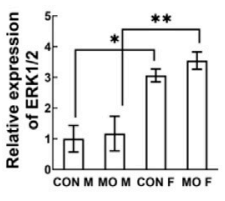

E

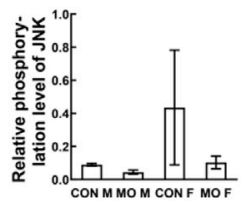

I

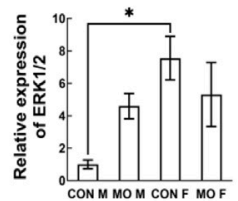

L

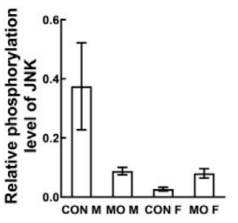

C

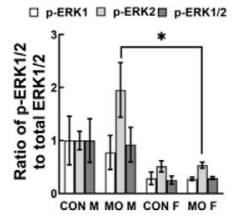

F



D



G



J

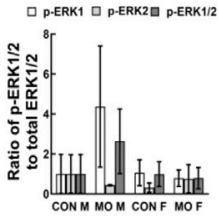

M

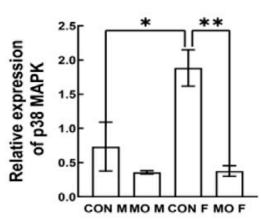

K

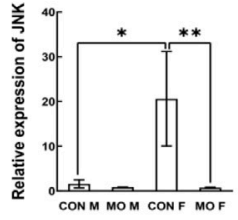

$\mathbf{N}$

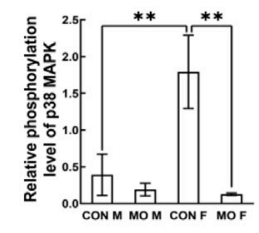

P

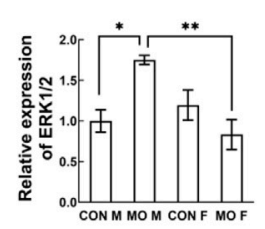

S

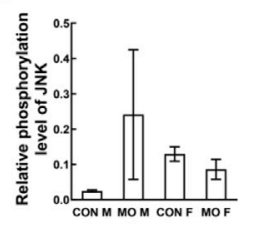

Q

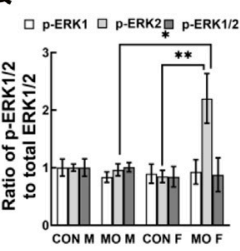

T

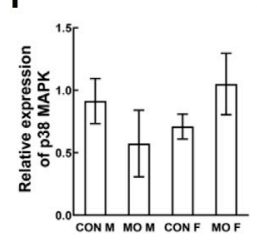

R

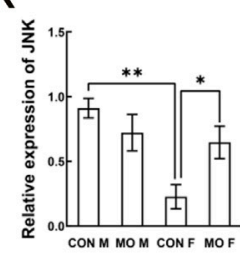

U

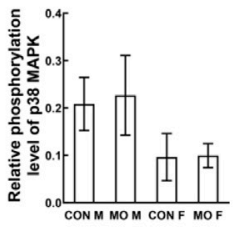

FIGURE 1 | Alteration of MAPK/ERK signaling pathway in offspring heart of overfed ewes at two gestational and one early postnatal time point. (A-G). Relative expression and phosphorylation level of hallmark proteins in fetal heart at mid-gestation day $90 ;(\mathbf{H}-\mathbf{N})$. Relative expression and phosphorylation level of hallmark proteins in fetal heart at late-gestation day 135; (O-U). Relative expression and phosphorylation level of hallmark proteins in neonatal heart at day 1 after birth; GAPDH, Protein loading control. CON, control, MO, maternal obesity, M, male, and F, female. Mean \pm SEM $(n=5) ;{ }^{*} p<0.05,{ }^{* *} p<0.01$. 
provided by the manufacturer). The membrane was blocked with $5 \%$ non-fat milk for 2 hours at room temperature (RT) and probed overnight at $4{ }^{\circ} \mathrm{C}$ with the following antibodies with dilution range from 1:500 to 1,500: LC3 rabbit antibody $(1: 1,000)$ (Catalog \# 2775S; Cell Signaling), SAPK/JNK rabbit antibody (1:500) (Catalog \# 9252S; Cell Signaling), anti-rabbit Phospho-SAPK/JNK rabbit antibody (1:1,000) (Catalog \# 4,668; Cell Signaling), phosphoAKT473 rabbit antibody (1:1,500) (Catalog \# 4060S; Cell Signaling), P38 MAPK rabbit antibody $(1: 1,000)$ (Catalog \# 8690S; Cell Signaling), phosphor-p38 MAPK rabbit antibody (1:


(1:1,000) (Catalog \# 50-172-9,292; Proteintech), TNFa rabbit antibody (1:1,000) (Catalog \# PBOTNFAI; Invitrogen), Atg5 rabbit antibody (1:1,500) (Catalog \# 12994S; Cell Signaling), AKT (pan) rabbit antibody (1:1,000) (Catalog \# 4691S; Cell Signaling), Rab7 rabbit antibody (1:1,500) (Catalog \# 9367S; Cell Signaling), anti-GAPDH rabbit mAB (1:1,000) (Catalog \# 2118S; Cell Signaling), p44/42 MAPK Horseradish Peroxidase (HRP) conjugated rabbit antibody (1:500) (Catalog \# 4348S; Cell Signaling), phosphor-p44/42 MAPK HRP conjugated rabbit antibody (1:1,000) (Catalog \# 8544S; Cell Signaling). HRP conjugated secondary antibodies were then incubated with antirabbit IgG $(1: 5,000)$ (Catalog \# 4,011; Promega corporation) for 1 hour at RT. Membranes were developed using the SuperSignal West Pico PLUS Chemiluminescent Substrate (Catalog \# 34,579; Thermo Scientific) and signals were obtained using ChemiDoc MP imaging system (Bio-Rad, Hercules, CA). The detailed procedure is found in our previous publication (Wang et al., 2019).

\section{Heart Tissue Cortisol Level Measurement}

Fifty mg of heart tissue from each group $(n=5)$ were lysed in PBS through homogenization with a Dounce homogenizer. The lysate was diluted 1:10 in diethyl ether and vortexed. The organic layer was recovered, dried under $\mathrm{N}$ gas and resuspended in cortisol ELISA buffer (Arbor Assays, \#K003). Cortisol was measured by ELISA following provider instructions (Arbor Assays, Ann Arbor, and MI) and previous publication (Stillo et al., 2021).

\section{Statistical Analysis}

Prism software (GraphPad, La Jolla, CA) was used for statistical analysis. Results were expressed as means \pm SEM. Statistical significance was determined with two-way ANOVA analysis of differences affected by two factors: treatment and sex at each time point. Bonferroni's multiple comparisons test were used to determine the significant differences between each type of treatment within same gender, or each gender within same treatment. Significance was set at values of $p<0.05$.

\section{RESULTS}

\section{Activation of MAPK Signaling in Fetal and Neonatal Sheep Hearts of Offspring of Maternal Obese Ewes}

Autophagy is an effective internal regulatory mechanism that allows biological organisms to adapt to different environments and protect organisms from metabolic stress (Levine and Kroemer, 2008). Either the enhancement or the suppression of autophagy is observed in obesity (Ignacio-Souza et al., 2014). Whether MO induces or suppresses autophagy flux in offspring tissues remains unclear. Studies have linked MAPK signaling with autophagy (Zhou et al., 2015; Zhang et al., 2018). To estimate alteration of autophagy associated signaling in heart tissue of fetuses and neonates of obese ewes, the family members involved in MAPK signaling, such as extracellular signal-regulated kinase (ERK)1/2, JNK, and p38 MAPK, were selected as hallmark proteins to be examined using western blotting. We first detected the protein expression of hallmark proteins at mid-gestation day 90 (GD90) in control and MO male and female fetal sheep hearts. The results showed that total ERK expression in control females was increased compared with control males $(p=0.0125)$. MO female fetuses also had increased total ERK expression compared with MO male fetuses ( $p=0.0058$ ) (Figures 1A,B). However, phosphorylation of ERK was not altered between groups $(p>0.08)$ except that pERK2 in MO female fetuses was increased compared with $\mathrm{MO}$ male fetuses $(p=0.0140$ ) (Figures 1A,C). JNK and p38 MAPK expression were not different between all groups $(p>0.09)$ (Figures 1A,D,F), however, the phosphorylation of JNK was increased in only control Females and the phosphorylation level of p38 MAPK in MO heart was higher by comparing to their respective control in both genders (Male $p=0.0306$; Female $p=$ 0.0038) (Figures 1A,E,G). At GD 135, total ERK had no difference between treatment group and control group (Male $p=0.1573$; Female $p=0.4874$ ). Sex differences were observed between control groups. Total ERK was increased in control females compared with control male ( $p=0.0125$ ) (Figures 1H,I). Phosphorylation of ERK had no changes between all groups $(p>0.05)$ (Figures 1H,J). Total JNK expression was reduced in MO female fetuses compared to control female fetuses $(p=0.0071)$, but not in MO male fetuses vs Control male fetuses $(p>0.05)$ (Figures 1H,K). However, JNK expression was significantly different in control females compared with control males $(p=0.0149)$ (Figures 1H,K). Phosphorylation ratio of JNK had no differences between each group in GD 135 fetal heart $(p>0.05)$ (Figures 1H,L). Both P38 MAPK expression and phosphorylation had no differences in $\mathrm{MO}$ vs control in male fetuses $(p>0.05)$, but were lower in MO female fetuses compared with control female fetuses (Expression $p=0.0033$ and Phosphorylation $p=0.0014$ ), and the female control was higher than that in male control (Expression $p=0.0385$ and phosphorylation $p=0.0092$ ) (Figures $\mathbf{1} \mathbf{M}, \mathbf{N}$ ). At dayl after birth, we found that total ERK was increased in MO male neonates compared with control male neonates $(p=0.0154)$ and MO female neonates $(p=0.0051)$ respectively (Figure 10,P). Phosphorylation of ERK2 was increased in MO female neonates compared with control female $(p=0.0064)$ and MO male neonates $(p=0.0105)$ respectively (Figure 1Q). JNK was increased in MO female neonates compared to control female neonates $(p=0.0427)$ and reduced in control female neonates compared to control male neonates ( $p=0.0014)$ (Figure 1R). Phosphorylation of JNK was not significantly different between each group $(p>0.05)$ (Figure 1S). No differences were found for the expression and phosphorylation of P38 MAPK between each group $(p>0.05)$ (Figure 1T,U). 
A
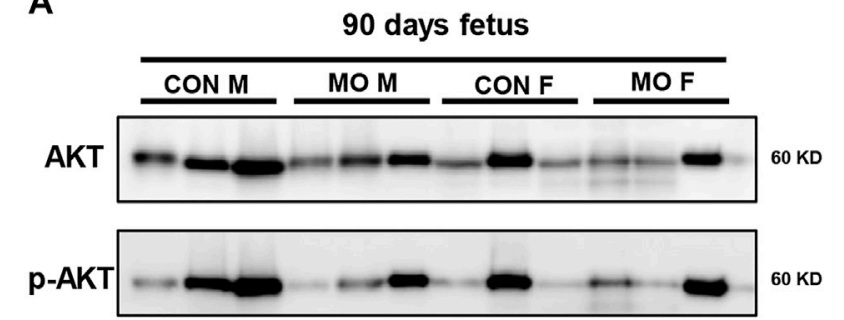

GAPDH

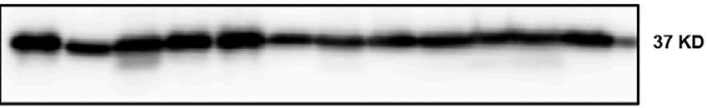

D

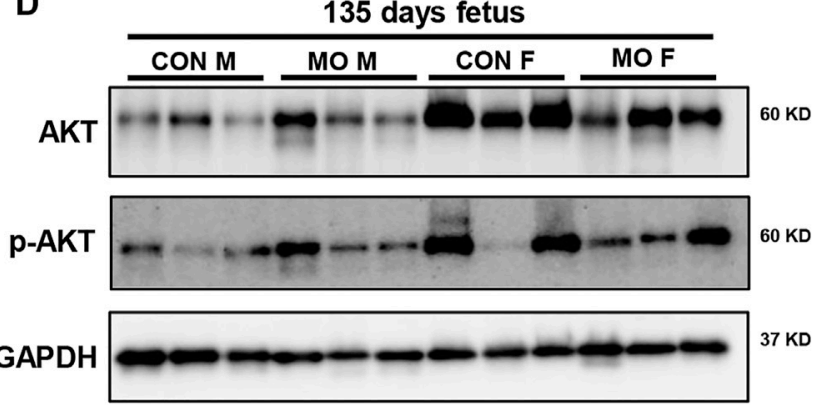

G Day 1 neonatal lamb

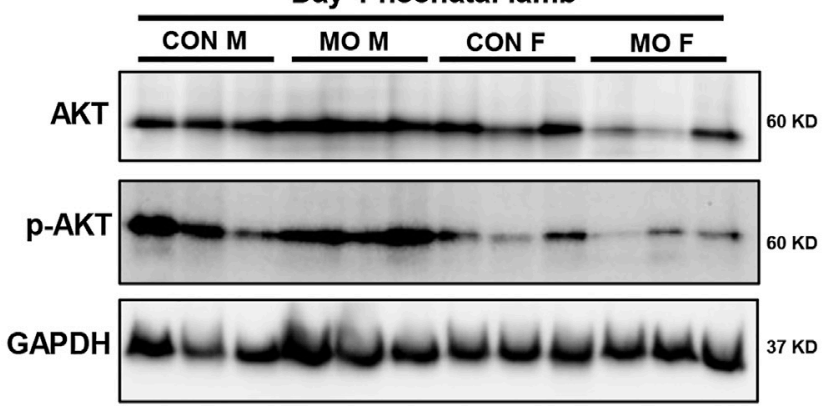

B

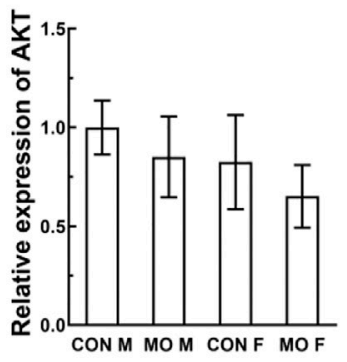

E

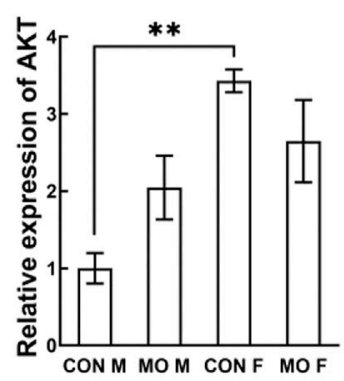

H



C

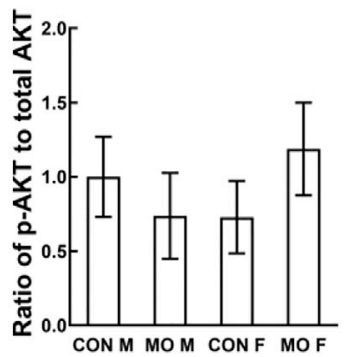

$\mathbf{F}$



I

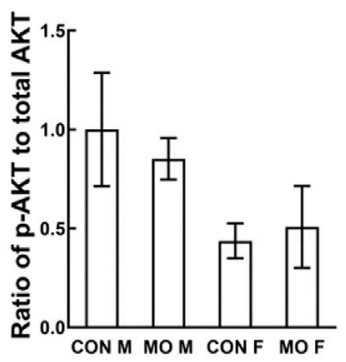

FIGURE 2 | Alteration of AKT signaling pathway in offspring heart of overfed ewes at two gestational and one early postnatal time point. (A-C). Relative expression and phosphorylation level of the kinase Akt in fetal heart at mid-gestation day 90; (D-F). Relative expression and phosphorylation level of the kinase Akt in fetal heart at late-gestation day 135; (G-I). Relative expression and phosphorylation level of the kinase Akt in neonatal heart at day 1 after birth; GAPDH, Protein loading control. CON, control, MO, maternal obesity, M, male, and F, female. Mean $\pm \operatorname{SEM}(n=5) ;{ }^{*} p<0.05,{ }^{* *} p<0.01$.

\section{Activation of the PI3K/AKT/mTOR Signaling Pathway in Overfed Male and Female Fetal and Neonatal Heart}

The kinase mammalian target of rapamycin (mTOR) is a major regulator of the autophagic process which is indirectly regulated by the survival PI3K/AKT pathway, the upstream of mTOR (Heras-Sandoval et al., 2014). We then detected expression level and activity of Akt in our overfed sheep model at DG90, DG135, and day 1 after birth. At DG90, we did not observe significant changes of Akt expression and phosphorylation level in MO groups compared with control groups nor in sex comparisons ( $p>0.05$ ) (Figures 2A-C). At DG135, Akt expression was only increased in control females compared with control males ( $p=0.0028)$, suggesting the protective role in female relative to male in response to maternal stress (Salminen et al., 2012; Ricke-Hoch et al., 2014). However, there was no difference between other treated and untreated groups $(p>0.05)$ (Figures 2D-F). At day 1 after birth, we observed decreased expression of Akt in MO female neonates compared with control female $(p=0.0202)$ and MO male neonates $(p=0.0153)$ respectively, and no differences were observed between any other groups $(p>0.05)$ (Figures 2G-I). 




C

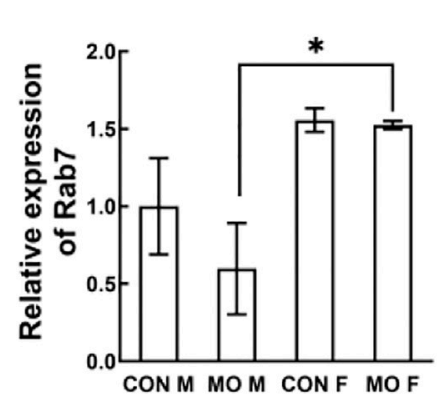

D



B



E

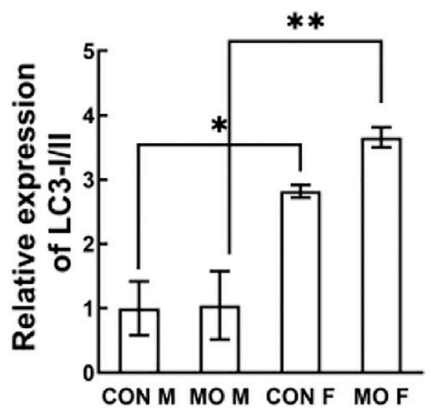

$\mathbf{F}$

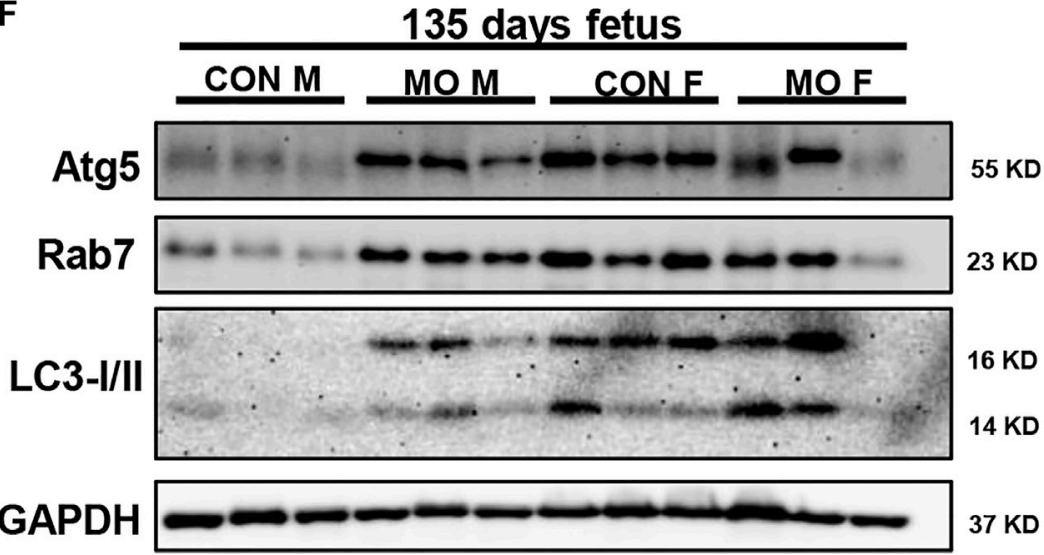

G

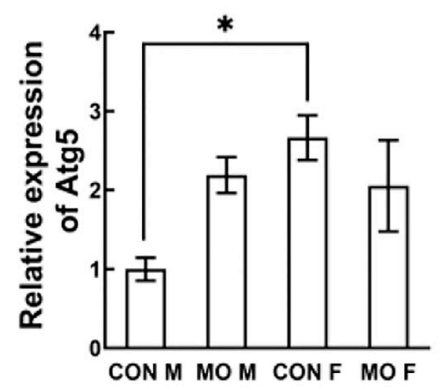

FIGURE 3 | Alteration of autophagy signaling pathway in offspring heart of overfed ewes at two gestational and one early postnatal time point. (A-E). Relative expression and phosphorylation level of hallmark proteins in fetal heart at mid-gestation day 90; (F-J). Relative expression and phosphorylation level of hallmark proteins in fetal heart at late-gestation day 135; (K-O). Relative expression and phosphorylation level of hallmark proteins in neonatal heart at day 1 after birth; GAPDH, Protein loading control. CON, control, MO, maternal obesity, M, male, and F, female. Mean $\pm \mathrm{SEM}(n=5) ;{ }^{\star} p<0.05$, ${ }^{\star \star} p<0.01$.

\section{Expression of the Autophagy-Related Proteins (ATGs) in Overfed Male and Female Fetal and Neonatal Hearts}

Autophagy is regulated by a number of signaling molecules, particularly the ATG family (Kim and Lee, 2014; Galluzzi et al., 2014;Galluzzi et al., 2017). Atg5 is considered an important ATG, as it is indispensable in both canonical and non-canonical autophagy (Bouderlique et al., 2016; Ye et al., 2018). Microtubule-associated protein 2 light chain 3 (LC3-II) and GTPase Rab7 are other common markers of autophagy in mammals (Tanida et al., 2004; González-Rodríguez et al., 2014; Kjos et al., 2017; Zheng et al., 2019). Because of their importance in the autophagy process and availability of antibodies that crossreact in sheep, we chose these autophagic hallmarks to indicate the changes of autophagic flux in the offspring of MO ewes. At 

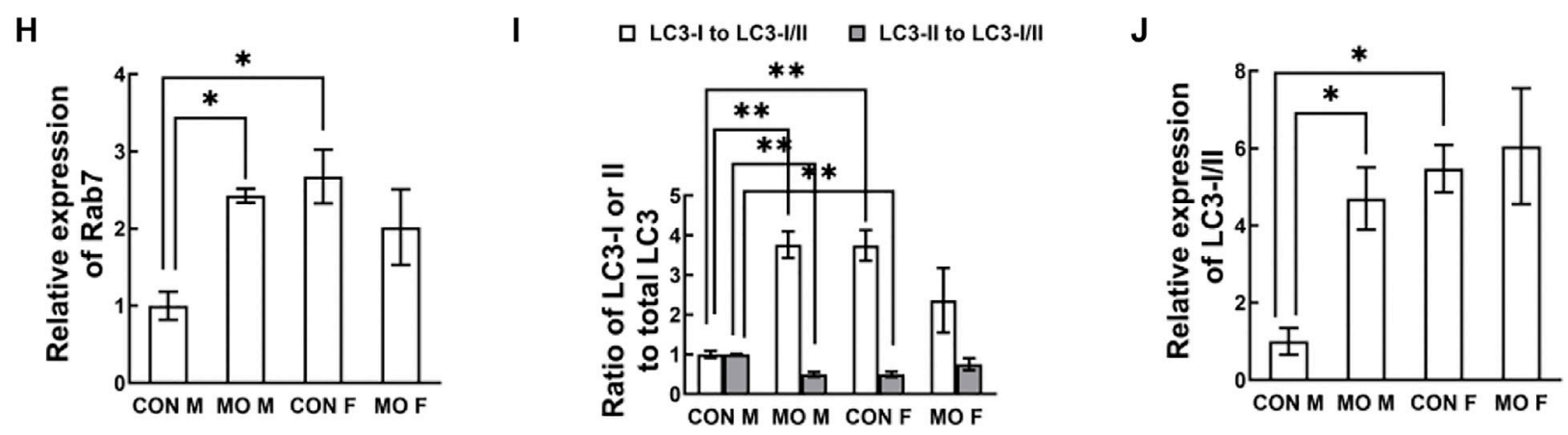

K

Day 1 neonatal lamb

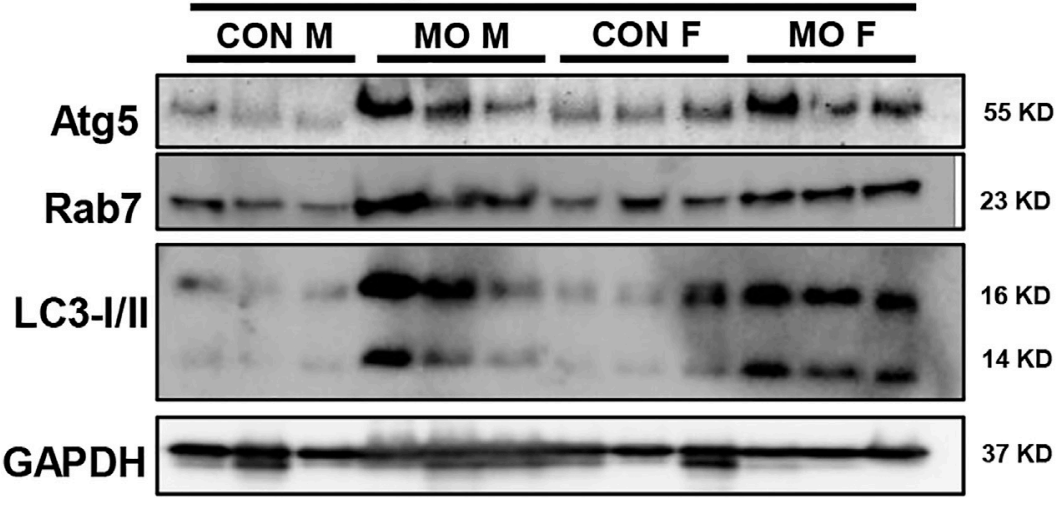

L

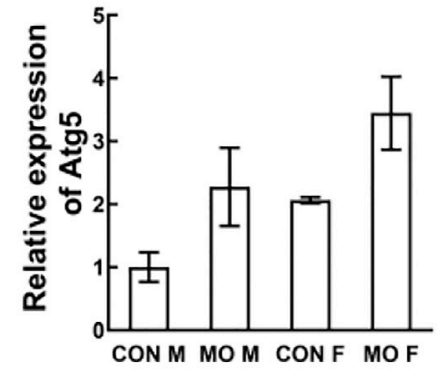

M

$\mathbf{N}$
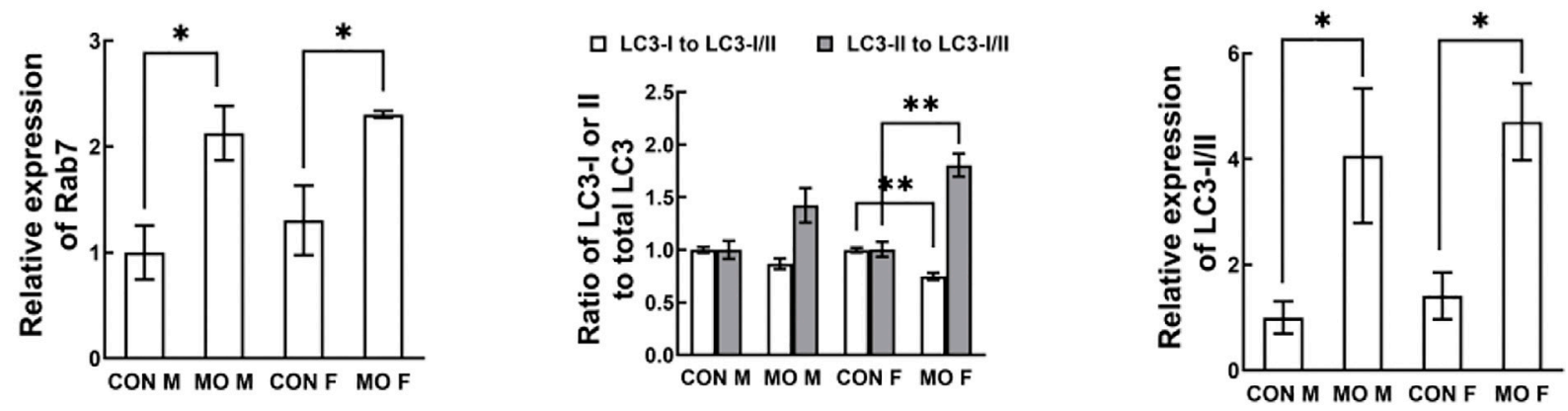

FIGURE 3 | (continued)

DG90, we found that there were no differences in Atg5, Rab7, and LC3-I/II between MO and control fetuses $(p>0.05)$, however, sex differences were observed (Atg5 of MO group $p=0.0313$; Rab7 of MO group $p=0.0335$; LC3-I/II of control group $p=0.0126$; LC3$\mathrm{I} / \mathrm{II}$ of MO group $p=0.0015)$ (Figures 3A-E). Atg5 $(p=0.0313)$ and Rab7 ( $p=0.0335)$ were increased in MO female fetuses compared with MO male fetuses (Figures 3B,C); Total LC3-I/II was increased in females compared with males between controls $(p=0.0126)$ and between MO fetuses $(p=0.0015)$ respectively (Figure 3E). At DG135, control female fetuses had the increased ATG5 $(p=0.0195)$, and Rab7 $(p=0.0115)$ when compared to control male fetuses (Figures $\mathbf{3 F}-\mathbf{H}$ ). The ratio of LC3-I to II was decreased in MO male fetuses compared to control male fetuses $(p=0.0074)$ and the decreased ratio was also observed in control female fetuses compared with control male fetuses $(p=0.0077)$ (Figures 3F,I). Total LC3-I/II was increased in MO male fetuses $(p=0.0437)$ and control female fetuses $(p=0.0178)$ compared to control male fetuses (Figure 3J). At day 1 after birth, Atg5 level had no differences between any groups $(p>0.05)$ (Figures 3K,L). Rab7 was increased in MO male neonates compared to control male neonates $(p=0.0233)$ and in MO female neonates compared with control female neonates $(p=0.0404)$ (Figures 3K,M). The 
A



B

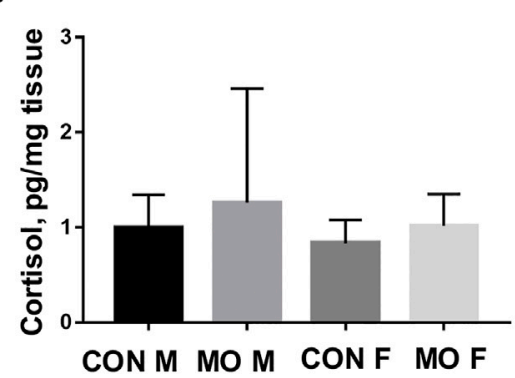

C

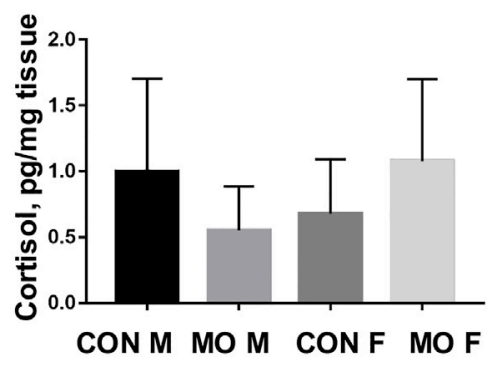

FIGURE 4 | Cortisol concentration in heart tissues of offspring of overfed ewes at two gestational and one early postnatal time point. (A) Cortisol concentration in fetal heart tissues at day 90 of gestation; (B) Cortisol concentration in fetal heart tissues at day 135 of gestation; (C) Cortisol concentration in neonatal heart tissues at day 1 after birth; CON, control, MO, maternal obesity, M, male, and F, female. Mean \pm SEM $(n=5)$. No significance.

ratio of LC3-I to II was increased in MO female neonates compared to control female neonates $(p=0.0021)$ (Figures $\mathbf{3 K}, \mathbf{N})$. Total LC3-I/II was increased in MO male neonates compared with control male neonates $(p=0.0482)$ and in $\mathrm{MO}$ female neonates compared with control female neonates $(p=$ 0.0350) (Figures 3K,O).

\section{Cortisol Concentration in Overfed Male and Female Fetal and Neonatal Heart}

Studies have shown that cortisol concentrations are elevated in MO mothers and fetuses at both mid- and late-gestation (Buss et al., 2012; Nathanielsz et al., 2013; Odhiambo et al., 2020). Growing evidence has suggested that excessive cortisol is associated with autophagy (Swerdlow et al., 2008; Harr et al., 2010; Ahn et al., 2014; Wnuk and Kajta, 2017). To evaluate whether cortisol concentrations are increased in MO fetal and neonatal hearts, we performed ELISA assays to detect cortisol concentrations at DG90, DG135, and day 1 after birth. The results indicated that there were no changes observed in the heart of overfed fetuses and neonatal sheep at three developmental stages (at DG90, $p=0.436$; at DG135, $p=0.491$ and at day $1, p=0.697$ ) (Figures 4A-C).

\section{Expression of Inflammatory Cytokines in Overfed Male and Female Fetal and Neonatal Hearts}

Autophagy can also be regulated by inflammation (Qian et al., 2017; Matsuzawa-Ishimoto et al., 2018). To further explore whether MO-induced autophagy in later developmental stage is associated with inflammation, the expression of inflammatory cytokines NF- $\kappa \mathrm{B}$ and $\mathrm{TNF} \alpha$ was determined. At DG90, the expression of $\mathrm{NK}-\kappa \mathrm{B}$ was significantly higher in $\mathrm{MO}$ male fetuses than that in control $(p=0.0075)$, but no difference was detected between $\mathrm{MO}$, and control female fetuses $(p>$ 0.05) (Figures 5A,B). The expression of TNFa in control male fetuses was significantly lower than that in MO male $(p=0.0263)$ and control female fetuses $(p=0.0084)$ (Figures 5A,C). However, the expression of both inflammatory cytokines was not different between each group in DG 135 fetuses and neonates $(p>0.05)$ (Figures 5D-I).

\section{DISCUSSION}

Poor maternal nutrition during gestation can alter lamb growth rates, tissue composition, and organ size at early postnatal time points (Godfrey and Barker, 2001; Hoffman et al., 2014; Reed et al., 2014; Hoffman et al., 2016; Raja et al., 2016). Our previous study showed that MO impairs fetal heart contractile function (Wang et al., 2019), which may be associated with changes in autophagy. However, little is known about the effects of poor maternal nutrition on heart muscle in the overfed sheep model. In this study, we further evaluated whether overfeeding during gestation affects autophagy associated pathways at two gestational and one early postnatal time point in male and female offspring. As expected, we observed altered expression of autophagy associated proteins in MAPK/ERK signaling, PI3K/ AKT/mTOR signaling and autophagy signaling pathways in both male and female offspring between $\mathrm{MO}$ and controls at late gestational stage (GD135) and early postnatal stage (day 1 after birth). Most intriguingly, our results suggest altered autophagy associated pathways exhibit sex differences. We found that female offspring were more profoundly influenced than male offspring by overfeeding of the dam during gestation.

Autophagy is a conserved process that catabolizes unnecessary and/or dysfunctional intracellular components for quality control to attenuate stress and maintain cellular homeostasis (Cheng et al., 2013; Sinha et al., 2017). The autophagic process is regulated by a number of signaling molecules, among which the mTOR kinase has a master role (Figure 6; Galluzzi et al., 2014; Galluzzi et al., 2017). The kinase mTOR induces phosphorylation of the autophagy-initiating ULK1 molecular complex and suppresses formation of autophagosome and autophagolysosome through a number of autophagy-related proteins including ATG5, LC3, and Rab7 (Figure 6; Ganley et al., 2009; Hosokawa et al., 2009; Jung et al., 2009). mTOR is a downstream target of the kinases PI3K and AKT (Manning and Cantley, 2007; Heras-Sandoval et al., 2014). Activated AKT can phosphorylate and activate mTOR and 

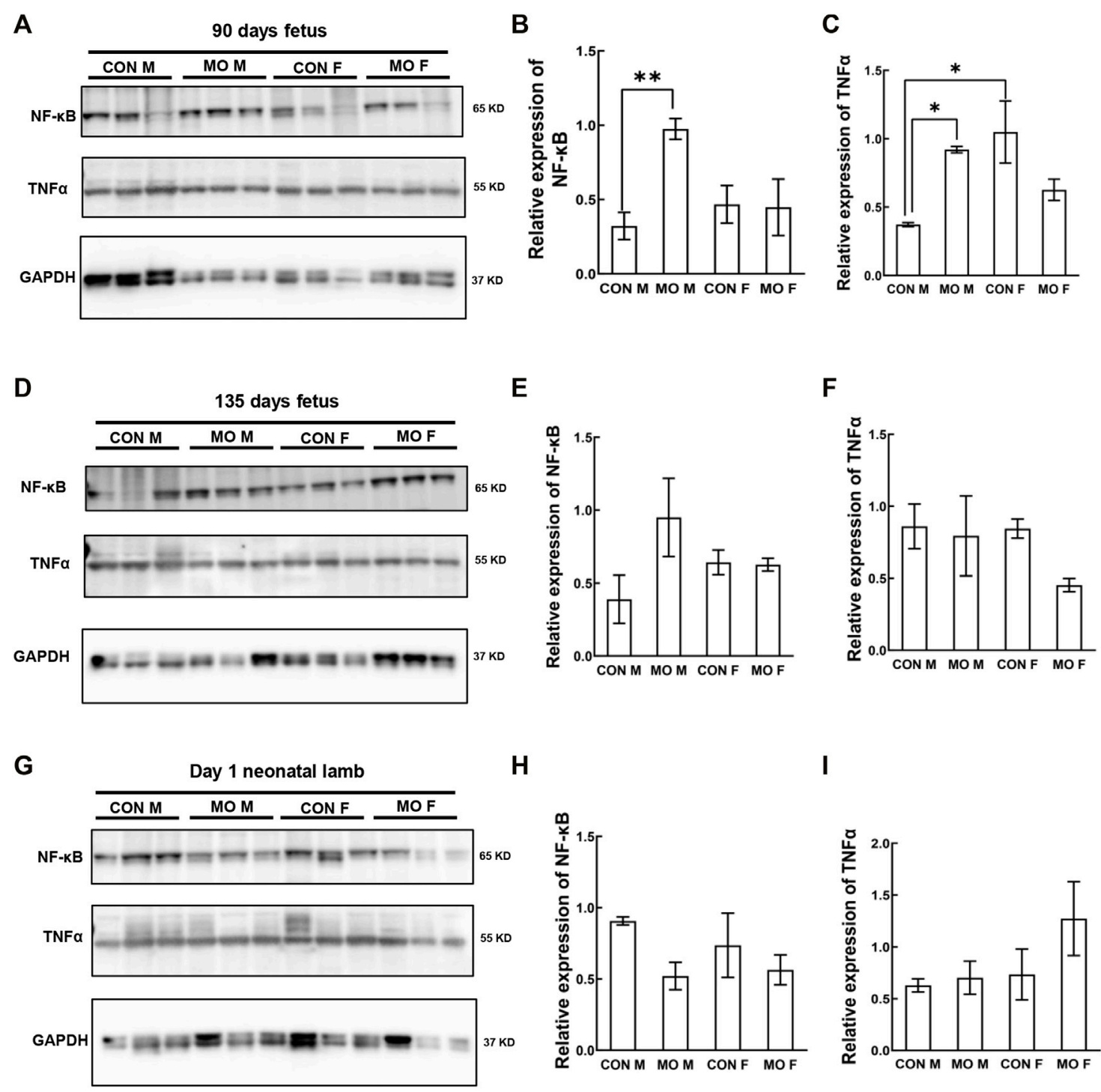

H

I
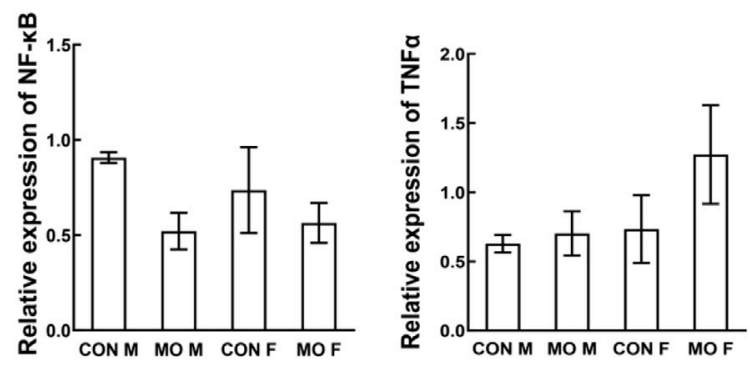

FIGURE 5 | Alteration of inflammatory cytokine NF-kB and TNFa in offspring heart of overfed ewes at two gestational and one early postnatal time point. (A-C). Relative expression level of inflammatory cytokine in fetal heart at mid-gestation day 90; (D-F). Relative expression level of inflammatory cytokine in fetal heart at lategestation day 135; (G-I). Relative expression level of inflammatory cytokine in neonatal heart at day 1 after birth; GAPDH, Protein loading control. CON, control, MO, maternal obesity, M, male, and F, female. Mean \pm SEM $(n=5) ;{ }^{*} p<0.05,{ }^{* *} p<0.01$.

thus suppress autophagy through ULK1/2 (Figure 6; Inoki et al., 2002; Saucedo et al., 2003; Menon et al., 2014). In addition, MAPKs, in particular p38 MAPK, activate mTOR in autophagy signaling. Furthermore, ERK and p38 MAPK regulate autophagy in response to various stimuli (Figure 6) (Webber, 2010). Recent studies show that JNK, one of MAPK subfamily members is also involved in the regulation of autophagy in response to environmental stress (Zhou et al., 2015). Examination of these signaling pathways that indirectly regulate autophagy through mTOR in overfed fetal and neonatal hearts demonstrated that overnutrition alters signaling that regulates autophagy in offspring of obese ewes (Figure 6). However, the effects are more overt in late gestation and early postnatal life. For example, at GD90, the autophagy associated proteins Atg5, Rab7, and LC3-I/II were not activated in overfed male or female offspring; however, at GD135 and day 1 after birth, these proteins were activated, suggesting altered autophagy, in both males and females. A handful of studies have shown that autophagy level is upregulated in response to extra- or intracellular stress signals such as starvation, nutrient signaling, energy balance, and stress signaling etc. ( $\mathrm{He}$ and Klionsky, 2009). Maternal obesity in sheep may lead to increased food intakes, low birth weight and energy balance regulation in late gestation and early postnatal life (Muhlhausler et al., 2006) which could explain why we observed the elevated autophagy pathway in late gestation and 


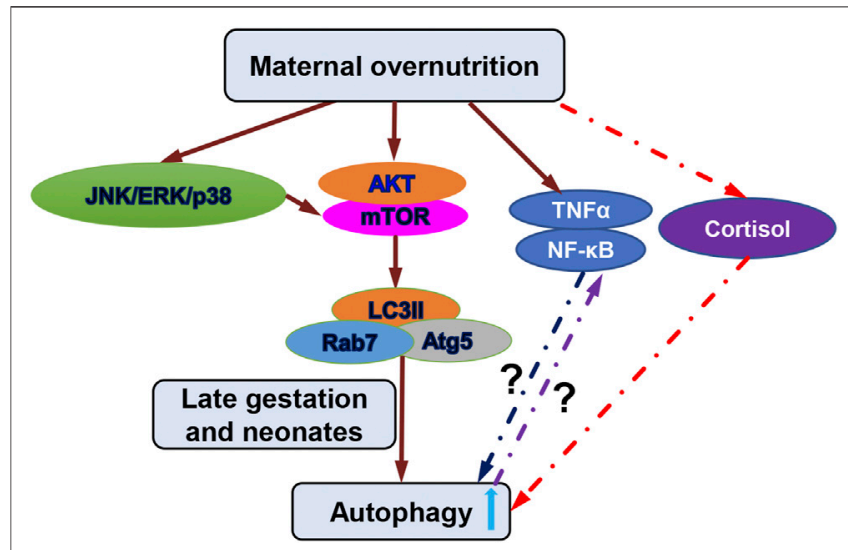

FIGURE 6 | Schematic diagram of autophagy associated signaling pathways and potential mechanisms regulated by maternal overnutrition. Important hallmark proteins in the PI3K/Akt/mTOR and the MAPK/ERK signaling are indicated which were examined in this study. Alternation of these hallmark proteins in later gestation and early postnatal life increased autophagy. Cortisol level did not change in heart tissues and was not the cause for the increased autophagy in later gestation and early postnatal life indicated by red dash line. Inflammatory factors TNF $\alpha$ and NF- $\mathrm{KB}$ were altered by maternal overnutrition in early gestation but not late gestation or early postnatal life in which the possible mechanisms could be that increased inflammatory factors in early gestation inhibits autophagy (blue dash line and question mark) and unchanged inflammatory factors at late gestation and early postnatal life could be suppressed by increased autophagy (purple dash line and question mark).

early postnatal life. Although the overall situation is more complicated, our data show that MO is a risk factor that may activate autophagy processes indirectly through upstream signaling of mTOR kinases in offspring heart muscles.

On the other hand, glucocorticoids have been linked to the induction of autophagy in response to stress in the cell such as nutrient deprivation (Juhász et al., 2003; Kinch et al., 2003; Kuma et al., 2004; Levine and Klionsky, 2004; Martin et al., 2007). Studies using under- and over-nutrition sheep model demonstrated that cortisol level was elevated in fetuses at the mid-term gestation and late-term gestation as well as in newborn lambs (Dong et al., 2008; Ford et al., 2009; Long et al., 2011; Zhang et al., 2011; Smith et al., 2018; Odhiambo et al., 2020). These studies suggest that autophagy associated signaling pathways could be induced by elevated cortisol level in fetal heart and neonatal hearts. However, our results revealed that MO fetal and neonatal hearts did not increase the cortisol level, implying that other potential mechanisms such as inflammation, may induce the altered autophagy associated protein expression. Inflammation is a common outcome of MO in many models (Moreli et al., 2012; Tarry-Adkins et al., 2016; Ghnenis et al., 2017; Jones et al., 2018; Montalvo-Martínez et al., 2018). Inflammation can regulate autophagy in both positive and negative ways (Trocoli and Djavaheri-Mergny, 2011; Salminen et al., 2012; Shi et al., 2016; Wu et al., 2016). We have tested the expression of two inflammatory factors TNFa and NF- $\kappa B$ in fetal and neonatal heart. The results showed that, compared to the control, the protein level of $\mathrm{NK}-\kappa \mathrm{B}$ and $\mathrm{TNFa}$ were all upregulated in GD90 MO male fetuses, but not in fetuses of later gestation stage (GD135) or neonates. Studies in sheep showed that TNFa or TNF super family member 11 were upregulated in the late gestation stage (GD135) or postnatal lambs (22-month-old) of overfed or obese ewes (Ghnenis et al., 2017). However, reports also showed that inflammation was not increased in fetal rat tissue in late gestation exposing to maternal obesity (Crew et al., 2016). Inflammatory response may vary in different organ and at different age of the offspring. In the present study, the sensitivity of inflammation to MO was opposite to that of autophagy at different developmental stages. Previous studies showed opposite trend of autophagy and inflammation in kidney (Nguyen et al., 2017; Sato et al., 2019), adipose tissue (Jansen et al., 2012), and placenta (Upadhyay et al., 2019) of offspring in response to maternal malnutrition. Considering the complex crosstalk between autophagy and inflammation (Harris et al., 2017; Deretic and Klionsky, 2018; Matsuzawa-Ishimoto et al., 2018), the possible mechanisms could be that 1) the inflammatory cytokines may inhibit the autophagy in early gestation stage, but were depressed by other factors in later gestation stage and after birth; or 2) the autophagy raised in the later stage inhibited the expression of inflammatory cytokines. The precise mechanisms by which the autophagy at the late stage of MO fetuses was induced in our study remain further investigation.

Lastly, effects of MO on the regulation of autophagy in other tissues or organs has been studied in animal models and human placenta. Studies using rodent models have reported that autophagy markers were suppressed in the kidneys of offspring of obese mothers with sex specificity (Nguyen et al., 2017). In a study with human placenta and mouse model, the researchers reported a sexual dimorphism in placental autophagy in response to MO (Muralimanoharan et al., 2016). Recent study in a MO sheep model demonstrated that autophagy protein markers were not altered in late-term MO F1 fetal livers (Serafim et al., 2021). The inconsistencies of altered and unaltered autophagy protein markers in different animal models and human studies indicate assessment of changes in autophagy with obesity can be rather complicated, as they depend on the nature, duration and models of obesity used, the tissue or cell types tested or simply the autophagy monitoring techniques used (Klionsky et al., 2016; Soussi et al., 2016; Zhang et al., 2018). Therefore, although our study supports that MO alters markers of autophagy in offspring hearts of obese ewes, future studies are required to determine if autophagy itself is altered and if these results can be transmitted to future generations.

\section{DATA AVAILABILITY STATEMENT}

The original contributions presented in the study are included in the article/Supplementary Material, further inquiries can be directed to the corresponding author.

\section{ETHICS STATEMENT}

The animal study was reviewed and approved by the University of Connecticut Institutional Animal Care and Use Committee. 


\section{AUTHOR CONTRIBUTIONS}

QD, YL, SA, SR, and WG designed the experiments; QD, YL, and $\mathrm{SH}$ performed the experiments; AJ, SP, MH, SR, KG, and SZ performed the animal study and tissue collections; QD, YL, SA, and WG analyzed and interpreted the data; QD and WG wrote the paper; SA, SR, and SZ revised the paper.

\section{REFERENCES}

Ahn, S., Park, J., An, I., Jung, S. J., and Hwang, J. (2014). Transient Receptor Potential Cation Channel V1 (TRPV1) Is Degraded by Starvation- and Glucocorticoid-Mediated Autophagy. Mol. Cell 37 (3), 257-263. doi:10.14348/molcells.2014.2384

Barker, D. J., and Clark, P. M. (1997). Fetal Undernutrition and Disease in Later Life. Rev. Reprod. 2 (2), 105-112. doi:10.1530/ror.0.0020105

Barker, D. J., Gluckman, P. D., Godfrey, K. M., Harding, J. E., Owens, J. A., and Robinson, J. S. (1993). Fetal Nutrition and Cardiovascular Disease in Adult Life. Lancet 341 (8850), 938-941. doi:10.1016/0140-6736(93)91224-a

Barker, D. J. (2007). The Origins of the Developmental Origins Theory. J. Intern. Med. 261 (5), 412-417. doi:10.1111/j.1365-2796.2007.01809.x

Bell, A. W., and Ehrhardt, R. A. (2002). Regulation of Placental Nutrient Transport and Implications for Fetal Growth. Nutr. Res. Rev. 15 (2), 211-230. doi:10.1079/ NRR200239

Boney, C. M., Verma, A., Tucker, R., and Vohr, B. R. (2005). Metabolic Syndrome in Childhood: Association with Birth Weight, Maternal Obesity, and Gestational Diabetes Mellitus. Pediatrics 115 (3), E290-E296. doi:10.1542/ peds.2004-1808

Bouderlique, T., Vuppalapati, K. K., Newton, P. T., Li, L., Barenius, B., and Chagin, A. S. (2016). Targeted Deletion of Atg5 in Chondrocytes Promotes Age-Related Osteoarthritis. Ann. Rheum. Dis. 75 (3), 627-631. doi:10.1136/annrheumdis2015-207742

Buss, C., Davis, E. P., Shahbaba, B., Pruessner, J. C., Head, K., and Sandman, C. A. (2012). Maternal Cortisol over the Course of Pregnancy and Subsequent Child Amygdala and hippocampus Volumes and Affective Problems. Proc. Natl. Acad. Sci. United States America 109 (20), E1312-E1319. doi:10.1073/ pnas. 1201295109

Caballero, E. (2003). Obesity, Diabetes, and the Metabolic Syndrome: New Challenges in Antipsychotic Drug Therapy. Cns Spectrums 8(11), 19-22. doi:10.1017/S1092852900008154

Cheng, Y., Ren, X. C., Hait, W. N., and Yang, J. M. (2013). Therapeutic Targeting of Autophagy in Disease: Biology and Pharmacology. Pharmacol. Rev. 65 (4), 1162-1197. doi:10.1124/pr.112.007120

Crew, R. C., Waddell, B. J., and Mark, P. J. (2016). Maternal Obesity Induced by a "Cafeteria" Diet in the Rat Does Not Increase Inflammation in Maternal, Placental or Fetal Tissues in Late Gestation. Placenta 39, 33-40. doi:10.1016/ j.placenta.2016.01.002

Deretic, V., and Klionsky, D. J. (2018). Autophagy and Inflammation: A Special Review Issue. Autophagy 14, 179-180. doi:10.1080/ 15548627.2017.1412229

Djavaheri-Mergny, M., Amelotti, M., Mathieu, J., Besançon, F., Bauvy, C., Souquère, S., et al. (2006). NF-kB Activation Represses Tumor Necrosis Factor- $\alpha$-Induced Autophagy. J. Biol. Chem. 281, 30373-30382. doi:10.1074/jbc.M602097200

Dong, F., Ford, S. P., Nijland, M. J., Nathanielsz, P. W., and Ren, J. (2008). Influence of Maternal Undernutrition and Overfeeding on Cardiac Ciliary Neurotrophic Factor Receptor and Ventricular Size in Fetal Sheep. J. Nutr. Biochem. 19 (6), 409-414. doi:10.1016/j.jnutbio.2007.06.003

Du, M., Huang, Y., Das, A. K., Yang, Q., Duarte, M. S., Dodson, M. V., et al. (2013). Meat Science and Muscle Biology Symposium: Manipulating Mesenchymal Progenitor Cell Differentiation to Optimize Performance and Carcass Value of Beef Cattle. J. Anim. Sci. 91 (3), 1419-1427. doi:10.2527/jas.2012-5670

Flegal, K. M., Carroll, M. D., Kit, B. K., and Ogden, C. L. (2012). Prevalence of Obesity and Trends in the Distribution of Body Mass Index Among US Adults, 1999-2010. Jama-Journal Am. Med. Assoc. 307 (5), 491-497. doi:10.1001/ jama.2012.39

\section{FUNDING}

This work was supported by the NIH HD101870, Wisconsin Alumni Research Foundation (AAH4884), University of Wisconsin Foundation (AAH5964), USDA-NIFA Hatch project (WIS04005), and USDA-NIFA 2014-01982.

Flegal, K. M., Carroll, M. D., Ogden, C. L., and Curtin, L. R. (2010). Prevalence and Trends in Obesity Among US Adults, 1999-2008. Jama-Journal Am. Med. Assoc. 303 (3), 235-241. doi:10.1001/jama.2009.2014

Ford, S. P., and Long, N. M. (2011). Evidence for Similar Changes in Offspring Phenotype Following Either Maternal Undernutrition or Overnutrition: Potential Impact on Fetal Epigenetic Mechanisms. Reprod. Fertil. Dev. 24 (1), 105-111. doi:10.1071/RD11911

Ford, S. P., Zhang, L. R., Zhu, M. J., Miller, M. M., Smith, D. T., Hess, B. W., et al. (2009). Maternal Obesity Accelerates Fetal Pancreatic $\beta$-cell but Not $\alpha$-cell Development in Sheep: Prenatal Consequences. Am. J. Physiology-Regulatory, Integr. Comp. Physiol. 297 (3), R835-R843. doi:10.1152/ajpregu.00072

Friedrich, M. J. (2002). Epidemic of Obesity Expands its Spread to Developing Countries. Jama-Journal Am. Med. Assoc. 287(11), 1382. doi:10.1001/ jama.287.11.1382

Gaillard, R. (2015). Maternal Obesity during Pregnancy and Cardiovascular Development and Disease in the Offspring. Eur. J. Epidemiol. 30 (11), 1141-1152. doi:10.1007/s10654-015-0085-7

Galluzzi, L., Bravo-San Pedro, J. M., Levine, B., Green, D. R., and Kroemer, G. (2017). Pharmacological Modulation of Autophagy: Therapeutic Potential and Persisting Obstacles. Nat. Rev. Drug Discov. 16 (7), 487-511. doi:10.1038/ nrd.2017.22

Galluzzi, L., Pietrocola, F., Levine, B., and Kroemer, G. (2014). Metabolic Control of Autophagy. Cell 159 (6), 1263-1276. doi:10.1016/j.cell.2014.11.006

Ganley, I. G., Lam, D. H., Wang, J. R., Ding, X. J., Chen, S., and Jiang, X. J. (2009). ULK1 center Dot ATG13 center Dot FIP200 Complex Mediates mTOR Signaling and Is Essential for Autophagy. J. Biol. Chem. 284 (18), 12297-12305. doi:10.1074/jbc.M900573200

Gauvin, M. C., Pillai, S. M., Reed, S. A., Stevens, J. R., Hoffman, M. L., Jones, A. K., et al. (2020). Poor Maternal Nutrition during Gestation in Sheep Alters Prenatal Muscle Growth and Development in Offspring. J. Anim. Sci. 98 (1). doi:10.1093/jas/skz388

Ghnenis, A. B., Odhiambo, J. F., McCormick, R. J., Nathanielsz, P. W., and Ford, S. P. (2017). Maternal Obesity in the Ewe Increases Cardiac Ventricular Expression of Glucocorticoid Receptors, Proinflammatory Cytokines and Fibrosis in Adult Male Offspring. PLoS One 12, 1-13. doi:10.1371/ journal.pone.0189977

Gluckman, P. D., Hanson, M. A., Cooper, C., and Thornburg, K. L. (2008). Effect of In Utero and Early-Life Conditions on Adult Health and Disease. N. Engl. J. Med. 359 (1), 61-73. doi:10.1056/NEJMra0708473

Godfrey, K. M., and Barker, D. J. (2001). Fetal Programming and Adult Health. Public Health Nutr. 4 (2B), 611-624. doi:10.1079/PHN2001145

González-Rodríguez, A., Mayoral, R., Agra, N., Valdecantos, M. P., Pardo, V., Miquilena-Colina, M. E., et al. (2014). Impaired Autophagic Flux Is Associated with Increased Endoplasmic Reticulum Stress during the Development of NAFLD. Cel Death Dis. 5 (4), e1179. doi:10.1038/Cddis.2014.162

Guo, W., Schafer, S., Greaser, M. L., Radke, M. H., Liss, M., Govindarajan, T., et al. (2012). RBM20, a Gene for Hereditary Cardiomyopathy, Regulates Titin Splicing. Nat. Med. 18 (5), 766-U162. doi:10.1038/nm.2693

Harr, M. W., McColl, K. S., Zhong, F., Molitoris, J. K., and Distelhorst, C. W. (2010). Glucocorticoids Downregulate Fyn and Inhibit IP3-Mediated Calcium Signaling to Promote Autophagy in T Lymphocytes. Autophagy 6 (7), 912-921. doi:10.4161/auto.6.7.13290

Harris, J., Lang, T., Thomas, J. P. W., Sukkar, M. B., Nabar, N. R., and Kehrl, J. H. (2017). Autophagy and Inflammasomes. Mol. Immunol. 86, 10-15. doi:10.1016/ j.molimm.2017.02.013

He, C., and Klionsky, D. J. (2009). Regulation Mechanisms and Signaling Pathways of Autophagy. Annu. Rev. Genet. 43, 67-93. doi:10.1146/annurev-genet102808-114910 
Heras-Sandoval, D., Perez-Rojas, J. M., Hernandez-Damian, J., and PedrazaChaverri, J. (2014). The Role of PI3K/AKT/mTOR Pathway in the Modulation of Autophagy and the Clearance of Protein Aggregates in Neurodegeneration. Cell Signal. 26 (12), 2694-2701. doi:10.1016/ j.cellsig.2014.08.019

Hoffman, M. L., Peck, K. N., Forella, M. E., Fox, A. R., Govoni, K. E., and Zinn, S. A. (2016). The Effects of Poor Maternal Nutrition on Postnatal Growth and Development of Lambs. J. Anim. Sci. 94 (2), 789-799. doi:10.2527/jas.2015-9933

Hoffman, M. L., Rokosa, M. A., Zinn, S. A., Hoagland, T. A., and Govoni, K. E. (2014). Poor Maternal Nutrition during Gestation in Sheep Reduces Circulating Concentrations of Insulin-like Growth Factor-I and Insulin-like Growth Factor Binding Protein-3 in Offspring. Domest. Anim. Endocrinol. 49, 39-48. doi:10.1016/j.domaniend.2014.05.002

Hosogi, S., Kusuzaki, K., Inui, T., Wang, X., and Marunaka, Y. (2014). Cytosolic Chloride Ion Is a Key Factor in Lysosomal Acidification and Function of Autophagy in Human Gastric Cancer Cell. J. Cel. Mol. Med. 18, 1124-1133. doi: $10.1111 / j \mathrm{cmm} .12257$

Hosokawa, N., Hara, T., Kaizuka, T., Kishi, C., Takamura, A., Miura, Y., et al. (2009). Nutrient-dependent mTORC1 Association with the ULK1-Atg13Fip200 Complex Required for Autophagy. Mol. Biol. Cel 20 (7), 1981-1991. doi:10.1091/mbc.E08-12-1248

Huang, Y., Yan, X., Zhao, J. X., Zhu, M. J., McCormick, R. J., Ford, S. P., et al. (2010). Maternal Obesity Induces Fibrosis in Fetal Myocardium of Sheep. Am.J. Physiol. Endocrinol. Metab. 299, E968-E975. doi:10.1152/ ajpendo.00434.2010

Ignacio-Souza, L. M., Bombassaro, B., Pascoal, L. B., Portovedo, M. A., Razolli, D. S., Coope, A., et al. (2014). Defective Regulation of the Ubiquitin/proteasome System in the Hypothalamus of Obese Male Mice. Endocrinology 155 (8), 2831-2844. doi:10.1210/en.2014-1090

Inoki, K., Li, Y., Zhu, T., Wu, J., and Guan, K. L. (2002). TSC2 Is Phosphorylated and Inhibited by Akt and Suppresses mTOR Signalling. Nat. Cel Biol 4 (9), 648-657. doi:10.1038/ncb839

Jansen, H. J., Van Essen, P., Koenen, T., Joosten, L. A. B., Netea, M. G., Tack, C. J., et al. (2012). Autophagy Activity Is Up-Regulated in Adipose Tissue of Obese Individuals and Modulates Proinflammatory Cytokine Expression. Endocrinology 153, 5866-5874. doi:10.1210/en.2012-1625

Jones, A. K., Hoffman, M. L., Pillai, S. M., McFadden, K. K., Govoni, K. E., Zinn, S. A., et al. (2018). Gestational Restricted- and Over-feeding Promote Maternal and Offspring Inflammatory Responses that Are Distinct and Dependent on Diet in Sheep. Biol. Reprod. 98, 184-196. doi:10.1093/biolre/iox174

Juhász, G., Csikós, G., Sinka, R., Erdélyi, M., and Sass, M. (2003). The Drosophila Homolog of Aut1 Is Essential for Autophagy and Development. FEBS Lett. 543 (1-3), 154-158. doi:10.1016/s0014-5793(03)00431-9

Jung, C. H., Jun, C. B., Ro, S. H., Kim, Y. M., Otto, N. M., Cao, J., et al. (2009). ULK-Atg13-FIP200 Complexes Mediate mTOR Signaling to the Autophagy Machinery. Mol. Biol. Cel 20 (7), 1992-2003. doi:10.1091/ mbc.E08-12-1249

Kim, K. H., and Lee, M. S. (2014). Autophagy-a Key Player in Cellular and Body Metabolism. Nat. Rev. Endocrinol. 10 (6), 322-337. doi:10.1038/nrendo.2014.35

Kinch, G., Hoffman, K. L., Rodrigues, E. M., Zee, M. C., and Weeks, J. C. (2003). Steroid-triggered Programmed Cell Death of a Motoneuron Is Autophagic and Involves Structural Changes in Mitochondria. J. Comp. Neurol. 457 (4), 384-403. doi:10.1002/cne.10563

Kjos, I., Distefano, M. B., Saetre, F., Repnik, U., Holland, P., Jones, A. T., et al. (2017). Rab7b Modulates Autophagic Flux by Interacting with Atg4B. Embo Rep. 18 (10), 1727-1739. doi:10.15252/embr.201744069

Klionsky, D. J., Abdelmohsen, K., Abe, A., Abedin, M. J., Abeliovich, H., Arozena, A. A., et al. (2016). Guidelines for the Use and Interpretation of Assays for Monitoring Autophagy (3rd Edition). Autophagy 12 (1), 1-222. doi:10.1080/ 15548627.2015.1100356

Kuma, A., Hatano, M., Matsui, M., Yamamoto, A., Nakaya, H., Yoshimori, T., et al. (2004). The Role of Autophagy during the Early Neonatal Starvation Period. Nature 23 (7020), 4321032-4321036. doi:10.1038/nature03029

Levine, B., and Klionsky, D. J. (2004). Development by Self-Digestion: Molecular Mechanisms and Biological Functions of Autophagy. Dev. Cel 6 (4), 463-477. doi:10.1016/s1534-5807(04)00099-1

Levine, B., and Kroemer, G. (2008). Autophagy in the Pathogenesis of Disease. Cell 132 (1), 27-42. doi:10.1016/j.cell.2007.12.018
Long, N. M., Ford, S. P., and Nathanielsz, P. W. (2011). Maternal Obesity Eliminates the Neonatal Lamb Plasma Leptin Peak. J. Physiol. 589 (Pt 6), 1455-1462. doi:10.1113/jphysiol.2010.201681

Malnick, S. D., and Knobler, H. (2006). The Medical Complications of Obesity. QJM 99 (9), 565-579. doi:10.1093/qjmed/hcl085

Manning, B. D., and Cantley, L. C. (2007). AKT/PKB Signaling: Navigating Downstream. Cell 129 (7), 1261-1274. doi:10.1016/j.cell.2007.06.009

Martin, D. E., Jones, A. K., Pillai, S. M., Hoffman, M. L., McFadden, K. K., Zinn, S. A., et al. (2019). Maternal Restricted- and Over-feeding during Gestation Result in Distinct Lipid and Amino Acid Metabolite Profiles in the Longissimus Muscle of the Offspring. Front. Physiol. 10. doi:10.3389/Fphys.2019.00515

Martin, D. N., Balgley, B., Dutta, S., Chen, J., Rudnick, P., Cranford, J., et al. (2007). Proteomic Analysis of Steroid-Triggered Autophagic Programmed Cell Death during Drosophila Development. Cell Death Differ 14 (5), 916-923. doi:10.1038/sj.cdd.4402098

Matsuzawa-Ishimoto, Y., Hwang, S., and Cadwell, K. (2018). Autophagy and Inflammation. Annu. Rev. Immunol. 36, 73-101. doi:10.1146/annurevimmunol-042617-053253

McMullen, S., Langley-Evans, S. C., Gambling, L., Lang, C., Swali, A., and McArdle, H. J. (2012). A Common Cause for a Common Phenotype: The Gatekeeper Hypothesis in Fetal Programming. Med. Hypotheses 78 (1), 88-94. doi:10.1016/ j.mehy.2011.09.047

Menon, S., Dibble, C. C., Talbott, G., Hoxhaj, G., Valvezan, A. J., Takahashi, H., et al. (2014). Spatial Control of the TSC Complex Integrates Insulin and Nutrient Regulation of mTORC1 at the Lysosome. Cell 156 (4), 771-785. doi:10.1016/j.cell.2013.11.049

Montalvo-Martínez, L., Maldonado-Ruiz, R., Cárdenas-Tueme, M., ReséndezPérez, D., and Camacho, A. (2018). Maternal Overnutrition Programs Central Inflammation and Addiction-like Behavior in Offspring. Biomed. Res. Int. doi:10.1155/2018/8061389

Moreli, J. B., Morceli, G., De Luca, A. K. C., Magalhães, C. G., Costa, R. A. A., Damasceno, D. C., et al. (2012). Influence of Maternal Hyperglycemia on IL-10 and TNF- $\alpha$ Production: The Relationship with Perinatal Outcomes. J. Clin. Immunol. 32, 604-610. doi:10.1007/s10875-011-9634-3

Muhlhausler, B. S., Adam, C. L., Findlay, P. A., Duffield, J. A., and McMillen, I. C. (2006). Increased Maternal Nutrition Alters Development of the AppetiteRegulating Network in the Brain. FASEB J. 20 (8), 1257-1259. doi:10.1096/fj.055241fje

Muralimanoharan, S., Gao, X. L., Weintraub, S., Myatt, L., and Maloyan, A. (2016). Sexual Dimorphism in Activation of Placental Autophagy in Obese Women with Evidence for Fetal Programming from a Placenta-specific Mouse Model. Autophagy 12 (5), 752-769. doi:10.1080/15548627.2016.1156822

Nathanielsz, P. W., Ford, S. P., Long, N. M., Vega, C. C., Reyes-Castro, L. A., and Zambrano, E. (2013). Interventions to Prevent Adverse Fetal Programming Due to Maternal Obesity during Pregnancy. Nutr. Rev. 71, S78-S87. doi:10.1111/nure. 12062

National Research Council (1985). Nutrient Requirements of Sheep. Washington, DC: National Academic Press.

Nguyen, L. T., Chen, H., Pollock, C., and Saad, S. (2017). SIRT1 Reduction Is Associated with Sex-specific Dysregulation of Renal Lipid Metabolism and Stress Responses in Offspring by Maternal High-Fat Diet. Sci. Rep. 7, 1-13. doi:10.1038/s41598-017-08694-4

Odhiambo, J. F., Pankey, C. L., Ghnenis, A. B., and Ford, S. P. (2020). A Review of Maternal Nutrition during Pregnancy and Impact on the Offspring through Development: Evidence from Animal Models of over- and Undernutrition. Int. J. Environ. Res. Public Health 17 (18). doi:10.3390/Ijerph17186926

Ogden, C. L., Carroll, M. D., McDowell, M. A., and Flegal, K. M. (2007). Obesity Among Adults in the United States -no Statistically Significant Chance since 2003-2004, 1-8.

Pillai, S. M., Jones, A. K., Hoffman, M. L., McFadden, K. K., Reed, S. A., Zinn, S. A., et al. (2017). Fetal and Organ Development at Gestational Days 45, 90, 135 and at Birth of Lambs Exposed to under- or Over-nutrition during Gestation. Translational Anim. Sci. 1 (1), 16-25. doi:10.2527/tas2016.0002

Qian, M., Fang, X., and Wang, X. (2017). Autophagy and Inflammation. Clin. Transl. Med. 6, 24. doi:10.1186/s40169-017-0154-5

Raja, J. S., Hoffman, M. L., Govoni, K. E., Zinn, S. A., and Reed, S. A. (2016). Restricted Maternal Nutrition Alters Myogenic Regulatory Factor Expression in Satellite Cells of Ovine Offspring. Anim, 1-4. doi:10.1017/S1751731116000070 
Reed, S. A., Raja, J. S., Hoffman, M. L., Zinn, S. A., and Govoni, K. E. (2014). Poor Maternal Nutrition Inhibits Muscle Development in Ovine Offspring. J. Anim. Sci. Biotechnol. 5 (1), 43. doi:10.1186/2049-1891-5-43

Reynolds, R. M., Allan, K. M., Raja, E. A., Bhattacharya, S., McNeill, G., Hannaford, P. C., et al. (2013). Maternal Obesity during Pregnancy and Premature Mortality from Cardiovascular Event in Adult Offspring: Follow-Up of 1323275 Person Years. Bmj-British Med. J. 347, F4539. doi:10.1136/Bmj

Ricke-Hoch, M., Bultmann, I., Stapel, B., Condorelli, G., Rinas, U., Sliwa, K., et al. (2014). Opposing Roles of Akt and STAT3 in the protection of the Maternal Heart from Peripartum Stress. Cardiovasc. Res. 101, 587-596. doi:10.1093/cvr/cvu010

Salminen, A., Hyttinen, J. M. T., Kauppinen, A., and Kaarniranta, K. (2012). Context-dependent Regulation of Autophagy by IKK-NF-Kb Signaling: Impact on the Aging Process. Int. J. Cel Biol. 2021, 849541. doi:10.1155/ $2012 / 849541$

Sato, S., Norikura, T., and Mukai, Y. (2019). Maternal Quercetin Intake during Lactation Attenuates Renal Inflammation and Modulates Autophagy Flux in High-Fructose-Diet-Fed Female Rat Offspring Exposed to Maternal Malnutrition. Food Funct. 10, 5018-5031. doi:10.1039/c9fo01134j

Saucedo, L. J., Gao, X., Chiarelli, D. A., Li, L., Pan, D., and Edgar, B. A. (2003). Rheb Promotes Cell Growth as a Component of the Insulin/TOR Signalling Network. Nat. Cel Biol 5 (6), 566-571. doi:10.1038/ncb996

Serafim, T. L., Cunha-Oliveira, T., Deus, C. M., Sardao, V. A., Cardoso, I. M., Yang, S. S., et al. (2021). Maternal Obesity in Sheep Impairs Foetal Hepatic Mitochondrial Respiratory Chain Capacity. Eur. J. Clin. Invest. 51 (2). doi:10.1111/eci.13375

Shi, L., Dong, N., Fang, X., and Wang, X. (2016). Regulatory Mechanisms of TGFB1-Induced Fibrogenesis of Human Alveolar Epithelial Cells. J. Cel. Mol. Med. 20, 2183-2193. doi:10.1111/jcmm.12918

Sinha, R. A., Singh, B. K., and Yen, P. M. (2017). Reciprocal Crosstalk between Autophagic and Endocrine Signaling in Metabolic Homeostasis. Endocr. Rev. 38 (1), 69-102. doi:10.1210/er.2016-1103

Smith, A. M., Pankey, C. L., Odhiambo, J. F., Ghnenis, A. B., Nathanielsz, P. W., and Ford, S. P. (2018). Rapid Communication: Reduced Maternal Nutrition during Early- to Mid-gestation Elevates Newborn Lamb Plasma Cortisol Concentrations and Eliminates the Neonatal Leptin Surge. J. Anim. Sci. 96 (7), 2640-2645. doi:10.1093/jas/sky215

Soussi, H., Clement, K., and Dugail, I. (2016). Adipose Tissue Autophagy Status in Obesity: Expression and Flux-Two Faces of the Picture. Autophagy 12 (3), 588-589. doi:10.1080/15548627.2015.1106667

Stillo, T., Norgard, R. J., and Stefanovski, D. (2021). The Effects of Solliquin Administration on the Activity and Fecal Cortisol Production of Shelter Dogs. J. Vet. Behav. 2021. doi:10.1016/j.jveb.2021.05.001

Sullivan, E. L., Smith, M. S., and Grove, K. L. (2011). Perinatal Exposure to HighFat Diet Programs Energy Balance, Metabolism and Behavior in Adulthood. Neuroendocrinology 93 (1), 1-8. doi:10.1159/000322038

Swerdlow, S., McColl, K., Rong, Y. P., Lam, M., Gupta, A., and Distelhorst, C. W. (2008). Apoptosis Inhibition by $\mathrm{Bcl}-2$ Gives Way to Autophagy in Glucocorticoid-Treated Lymphocytes. Autophagy 4(5), 612-620. doi:10.4161/ Auto.5920

Tanida, I., Ueno, T., and Kominami, E. (2004). LC3 Conjugation System in Mammalian Autophagy. Int. J. Biochem. Cel Biol. 36 (12), 2503-2518. doi:10.1016/j.biocel.2004.05.009

Tarry-Adkins, J. L., Fernandez-Twinn, D. S., Hargreaves, I. P., Neergheen, V., Aiken, C. E., Martin-Gronert, M. S., et al. (2016). Coenzyme Q10 Prevents Hepatic Fibrosis, Inflammation, and Oxidative Stress in a Male Rat Model of Poor Maternal Nutrition and Accelerated Postnatal Growth. Am. J. Clin. Nutr. 103, 579-588. doi:10.3945/ajcn.115.119834

Trocoli, A., and Djavaheri-Mergny, M. (2011). The Complex Interplay between Autophagy and NF-Kb Signaling Pathways in Cancer Cells. Am. J. Cancer Res. 1, 629-649. Available at: http://www.ncbi.nlm.nih. gov/pubmed/21994903\%0Ahttp://www.pubmedcentral.nih.gov/ articlerender.fcgi?artid=PMC3189824.

Upadhyay, A., Anjum, B., Godbole, N. M., Rajak, S., Shukla, P., Tiwari, S., et al. (2019). Time-restricted Feeding Reduces High-Fat Diet Associated Placental
Inflammation and Limits Adverse Effects on Fetal Organ Development. Biochem. Biophys. Res. Commun. 514, 415-421. doi:10.1016/j.bbrc.2019.04.154

Wallace, J. M., Bourke, D. A., Aitken, R. P., Milne, J. S., and Hay, W. W., Jr. (2003). Placental Glucose Transport in Growth-Restricted Pregnancies Induced by Overnourishing Adolescent Sheep. J. Physiol. 547 (Pt 1), 85-94. doi:10.1113/ jphysiol.2002.023333

Wang, J. Y., Ma, H., Tong, C., Zhang, H. Y., Lawlis, G. B., Li, Y. D., et al. (2010). Overnutrition and Maternal Obesity in Sheep Pregnancy Alter the JNK-IRS-1 Signaling Cascades and Cardiac Function in the Fetal Heart. Faseb J. 24 (6), 2066-2076. doi:10.1096/fj.09-142315

Wang, Q. R., Zhu, C. Q., Sun, M. M., Maimaiti, R., Ford, S. P., Nathanielsz, P. W., et al. (2019). Maternal Obesity Impairs Fetal Cardiomyocyte Contractile Function in Sheep. Faseb J. 33 (2), 2587-2598. doi:10.1096/fj.201800988R

Webber, J. L. (2010). Regulation of Autophagy by P38alpha MAPK. Autophagy 6 (2), 292-293. doi:10.4161/auto.6.2.11128

Widdowson, E. M., and McCance, R. A. (1975). A Review: New Thoughts on Growth. Pediatr. Res. 9 (3), 154-156. doi:10.1203/00006450-197503000-00010

Wnuk, A., and Kajta, M. (2017). Steroid and Xenobiotic Receptor Signalling in Apoptosis and Autophagy of the Nervous System. Int. J. Mol. Sci. 18 (11). doi:10.3390/Ijms18112394

Wu, G., Bazer, F. W., Wallace, J. M., and Spencer, T. E. (2006). Boardinvited Review: Intrauterine Growth Retardation: Implications for the Animal Sciences. J. Anim. Sci. 84 (9), 2316-2337. doi:10.2527/jas.2006-156

$\mathrm{Wu}, \mathrm{T} . \mathrm{T} ., \mathrm{Li}, \mathrm{W} . \mathrm{M}$. , and Yao, Y. M. (2016). Interactions between Autophagy and Inhibitory Cytokines. Int. J. Biol. Sci. 12, 884-897. doi:10.7150/ijbs.15194

Ye, X., Zhou, X. J., and Zhang, H. (2018). Exploring the Role of Autophagy-Related Gene 5 (ATG5) Yields Important Insights into Autophagy in Autoimmune/ Autoinflammatory Diseases. Front. Immunol. 9, 2334. doi:10.3389/ fimmu.2018.02334

Zambrano, E., and Nathanielsz, P. W. (2013). Mechanisms by Which Maternal Obesity Programs Offspring for Obesity: Evidence from Animal Studies. Nutr. Rev. 71, S42-S54. doi:10.1111/nure.12068

Zhang, L., Long, N. M., Hein, S. M., Ma, Y., Nathanielsz, P. W., and Ford, S. P. (2011). Maternal Obesity in Ewes Results in Reduced Fetal Pancreatic $\beta$-cell Numbers in Late Gestation and Decreased Circulating Insulin Concentration at Term. Domest. Anim. Endocrinol. 40 (1), 30-39. doi:10.1016/ j.domaniend.2010.08.004

Zhang, Y. M., Sowers, J. R., and Ren, J. (2018). Targeting Autophagy in Obesity: from Pathophysiology to Management. Nat. Rev. Endocrinol. 14 (6), 356-376. doi:10.1038/s41574-018-0009-1

Zheng, W., Xie, W. W., Yin, D. Y., Luo, R., Liu, M., and Guo, F. J. (2019). ATG5 and ATG7 Induced Autophagy Interplays with UPR via PERK Signaling. Cell Commun. Signaling 17, 42. doi:10.1186/s12964-019-0353-3

Zhou, Y. Y., Li, Y., Jiang, W. Q., and Zhou, L. F. (2015). MAPK/JNK Signalling: a Potential Autophagy Regulation Pathway. Biosci. Rep. 35, e00119. doi:10.1042/ BSR20140141

Conflict of Interest: The authors declare that the research was conducted in the absence of any commercial or financial relationships that could be construed as a potential conflict of interest.

Publisher's Note: All claims expressed in this article are solely those of the authors and do not necessarily represent those of their affiliated organizations, or those of the publisher, the editors and the reviewers. Any product that may be evaluated in this article, or claim that may be made by its manufacturer, is not guaranteed or endorsed by the publisher.

Copyright $\odot 2022$ Liu, Ding, Halderson, Arriola Apelo, Jones, Pillai, Hoffman, Reed, Govoni, Zinn and Guo. This is an open-access article distributed under the terms of the Creative Commons Attribution License (CC BY). The use, distribution or reproduction in other forums is permitted, provided the original author(s) and the copyright owner(s) are credited and that the original publication in this journal is cited, in accordance with accepted academic practice. No use, distribution or reproduction is permitted which does not comply with these terms. 